\title{
NRES: the Network of Robotic Echelle Spectrographs
}

\author{
Robert J. Siverd ${ }^{\mathrm{a}}$, Timothy M. Brown ${ }^{\mathrm{a}}$, John. Hygelund ${ }^{\mathrm{a}}$, Todd Henderson ${ }^{\mathrm{a}}$, Joseph R. Tufts ${ }^{\mathrm{a}}$, \\ Jason D. Eastman ${ }^{\mathrm{b}}$, Julian van Eyken ${ }^{\mathrm{c}}$, and Stuart Barnes ${ }^{\mathrm{d}}$ \\ ${ }^{a}$ Las Cumbres Observatory Global Telescope Network, 6740 Cortona Dr., Ste 102, Goleta, CA \\ 93117, USA \\ ${ }^{\mathrm{b}}$ Harvard-Smithsonian Center for Astrophysics, Cambridge, MA 02138, USA \\ ${ }^{\mathrm{c}}$ NASA Exoplanet Science Institute, California Institute of Technology, 770 South Wilson \\ Avenue, M/S 100-22, Pasadena, CA 91125, USA \\ dStuart Barnes Optical Design, 1094 NK, Amsterdam, Netherlands
}

\begin{abstract}
Las Cumbres Observatory Global Network (LCOGT) is building the Network of Robotic Echelle Spectrographs (NRES), which will consist of six identical, optical $(390-860 \mathrm{~nm}$ ) high-precision spectrographs, each fiber-fed simultaneously by up to two 1-meter telescopes and a thorium argon calibration source. We plan to install one at up to 6 observatory sites in the Northern and Southern hemispheres, creating a single, globally-distributed, autonomous spectrograph facility using up to twelve 1-meter telescopes. Simulations suggest we will achieve long-term radial velocity precision of $3 \mathrm{~m} / \mathrm{s}$ in less than an hour for stars brighter than $\mathrm{V}=12$. We have been funded with NSF MRI and ATI grants, and expect our first spectrograph to be deployed in fall 2016, with the full network operation of 5 or 6 units beginning in 2017. We will briefly overview the NRES design, goals, robotic operation, and status. In addition, we will discuss early results from our prototype spectrograph, the laboratory and on-sky performance of our first production unit, and the ongoing software development effort to bring this resource online.
\end{abstract}

Keywords: LCOGT, NRES, echelle, fiber, exoplanet spectrograph, radial velocity, robotic

\section{INTRODUCTION}

The Las Cumbres Observatory Global Telescope (LCOGT) Network has successfully deployed and now operates a worldwide network of research-grade optical/NIR telescopes across the world. As of today (May 2016), the network comprises two 2-meter telescopes, nine 1-meter telescopes, and seven 0.4-meter telescopes in both the Northern and Southern hemispheres with additional sites under construction (see Figure 1).

All telescopes are equipped with imagers and numerous photometric filters. Additionally, the 2-meter telescopes are equipped with low resolution slit spectrographs. For more information about the telescopes, mission, network operation, and software development, see reference 1. For a summary of the first two years in operation, see reference 2 .

\subsection{Exoplanets and Spectroscopy}

The recent explosive growth of exoplanet discovery and characterization has significantly increased the need for high-resolution spectroscopy. Recent years have seen a very rapid increase in the number of known exoplanets, with over 3000 known as of this writing ${ }^{3}$ and more in the offing. As predicted by the Astro2010 report, instruments capable of stellar classification and high-precision radial velocity (RV) of relatively bright stars $(V \leq 12)$ are in increasingly high demand as a result.

For transiting exoplanet surveys specifically (such as SuperWASP, ${ }^{4}$ HATNet, $^{5}$ and $\mathrm{KELT}^{6}$ ), the need for spectroscopy is two-fold. Firstly, spectroscopic observations of candidate host stars are used to identify and eliminate astrophysical false positives such as eclipsing binaries. Secondly, stellar radial velocity (RV) measurements

Further author information: (Send correspondence to R.J.S.)

R.J.S.: E-mail: rsiverd@lcogt.net, Telephone: 18058801633

Ground-based and Airborne Instrumentation for Astronomy VI, edited by Christopher J. Evans, Luc Simard, Hideki Takami Proc. of SPIE Vol. 9908, 99086X · C 2016 SPIE · CCC code: 0277-786X/16/\$18 · doi: 10.1117/12.2233188 
are used to extract orbital elements and infer the masses of the host star and its companion(s). This process may be further complicated by the need to sample the orbit if its period is close to 24 hours. Even for fairly bright stars, this is a time-consuming endeavor. Additionally, long-term RV monitoring is also able to uncover additional planets at longer periods.

NASA's forthcoming Transiting Exoplanet Survey Satellite (TESS) mission ${ }^{7}$ will conduct a full-sky survey of bright $(V \leq 12)$ stars for new transiting exoplanets and will have similar spectroscopic needs, exacerbating the growing shortage of time for high-precision spectroscopy. Follow-up of TESS targets will be crucial in order to identify the best candidates for detailed study by future space missions such as JWST.

\subsection{Network Advantages and Current Status}

LCOGT has developed the Network of Robotic Echelle Spectrographs (NRES) to meet this growing need. NRES consists of six identical, fiber-fed, temperature-controlled, cross-dispersed echelle spectrographs attached to LCOGT's 1-meter telescopes. Our simple optical design permits high optical throughput (up to 20\%) over a wide wavelength range $(390-860 \mathrm{~nm})$ and will deliver RV precision of $\sim 3 \mathrm{~m} / \mathrm{s}$. More detail about the optical system is provided in $\S 2$ and in Figure 2.

The resulting network will function as a single instrument for highly efficient high-resolution spectroscopy. In terms of RV measurements per hour, the full NRES network will outperform Keck/HIRES at $3 \mathrm{~m} / \mathrm{s}$ for stars brighter than $V \sim 12.5$ and at $30 \mathrm{~m} / \mathrm{s}$ for stars brighter than $V \sim 15 .^{8}$

The distributed approach confers other advantages as well. Most importantly, spectrographs at diverse sites provide weather redundancy, 24-hour availability and thus the ability to follow individual objects from site to site (weather permitting).

Since 2012, we have operated and tested a reduced-resolution $(R \sim 25000)$ prototype spectrograph on a $0.8 \mathrm{~m}$ telescope at our test site in Santa Ynez, CA. Critically, this prototype helped us find and fix design problems and also provided a platform to develop the new control software necessary to operate a fiber-fed spectrograph on our network.

Construction is nearing completion for the first production NRES unit and deployment is scheduled for fall 2016. At least three (up to five) additional units will be deployed during 2017, depending on initial deployment success and then site availability. Software for hardware control and data reduction are in active development to facilitate testing, deployment, and commissioning.

\section{SPECTROGRAPH DESIGN AND OPERATION}

NRES is a cross-dispersed echelle spectrograph with double-pass design (see Fig. 2). Dispersion and crossdispersion are performed by an R4 grating and 55-degree prism, respectively. Light enters the instrument through 67 -micron ( 2.75 arcsec diameter on sky) octagonal optical fibers and is then reflected by a turning mirror into the collimator and towards the dispersive elements. After dispersion, the light makes a second pass through the collimator, this time focusing it onto the CCD. The sensor is a $60 \mathrm{~mm} 4 \mathrm{~K} \mathrm{x} 4 \mathrm{~K}$ Fairchild $486 \mathrm{CCD}$ with 15-micron pixels. The resulting resolution is $R \sim 53000$ over a wide wavelength range $(390-860 \mathrm{~nm})$ with peak optical throughput of $\sim 20 \%$. For a more detailed description of the optical design, see reference 8 .

A small amount of light $(\sim 0.5 \%)$ is reflected off the initial prism surface. A mirror and spotting scope collect this stray light and feed it to an exposure meter operating in near-real-time. Tracking the flux on each fiber with few-second cadence will allow us to prevent overexposure, truncate exposures based on a target signal-to-noise ratio, and produce true flux-weighted time stamps in our analysis.

Each NRES unit accepts light through a three-fiber cable. Optical fibers into the spectrograph are octagonal with 67-micron diameter. The middle fiber connects to a calibration unit with a Thorium Argon (ThAr) lamp for wavelength calibration and a Tungsten Halogen lamp for flat-fielding. Each of the two "star fibers" carries starlight to the spectrograph from one of the 1-meter telescopes through its attached Acquisition and Guiding Unit (AGU).

In the focal plane, our 67 -micron fibers subtend $\sim 2.75$ arcseconds on the sky. This is larger than the typical seeing disc at our sites, ensuring that high throughput is maintained under most conditions. Assuming 




Figure 1. LCOGT network map depicting current and future telescope sites. Texas, Chile, South Africa, and Australia currently have operational 1-meter telescopes that will host NRES spectrographs. Additional sites in Israel and Tibet are under construction and will also host NRES spectrographs once incorporated into the network. The first unit will be deployed to Chile (CTIO) in fall 2016.

a Gaussian PSF, our fiber diameter collects $\sim 99.5 \%, \sim 96.5 \%$, and $\sim 90.2 \%$ of starlight in 2-, 2.5- and 3-arcsec seeing, respectively.

Simultaneous wavelength calibration is performed for all science images using ThAr lamp light on a dedicated calibration fiber adjacent to the science fiber. Fiber-to-fiber differences in wavelength solution are measured using special "double" calibration spectra with the same ThAr light simultaneously collected on two adjacent fibers. Using a separate fiber for wavelength calibration introduces some slight errors to the measurement, but these should be negligible if the instrument temperature and pressure are held to our specified stability level.

\section{ACQUISITION AND GUIDING UNIT}

The Acquisition and Guiding Unit (AGU) is installed on each NRES-connected telescope. It is the interface between the telescope (or lamps) and science fiber for both science and calibration data collection. During science observations, the AGU provides a view of the sky to identify the target, position it onto the science fiber, and make fine guide adjustments during the exposure. For calibration, the AGU redirects spectrograph lamp light into the science fiber for flats and tracing (with Tungsten Halogen) or fiber-to-fiber wavelength calibration (with ThAr).

Optically, the AGU consists of a curved mirror with a 225-micron pinhole bored through its center. The science fiber is affixed behind this pinhole. Light that passes through the pinhole is reimaged onto the fiber by a lens and carried to the spectrograph. Light that misses the pinhole reflects off the mirror to a camera lens that produces an unvignetted 8' x 12' image on the acquisition camera (Atik 460EX). The resulting image appears as normal sky with a black hole in the center (see Figure 3).

Robotic operation of a fiber-fed spectrograph on the LCOGT network requires development of new control software and lots of algorithmic fine-tuning, particularly for guiding and acquisition. In addition to vetting our optical design (and inspiring numerous improvements), the prototype NRES spectrograph has been a critical resource for identifying new development tasks and testing new algorithms. Acquisition and guiding, in particular, 

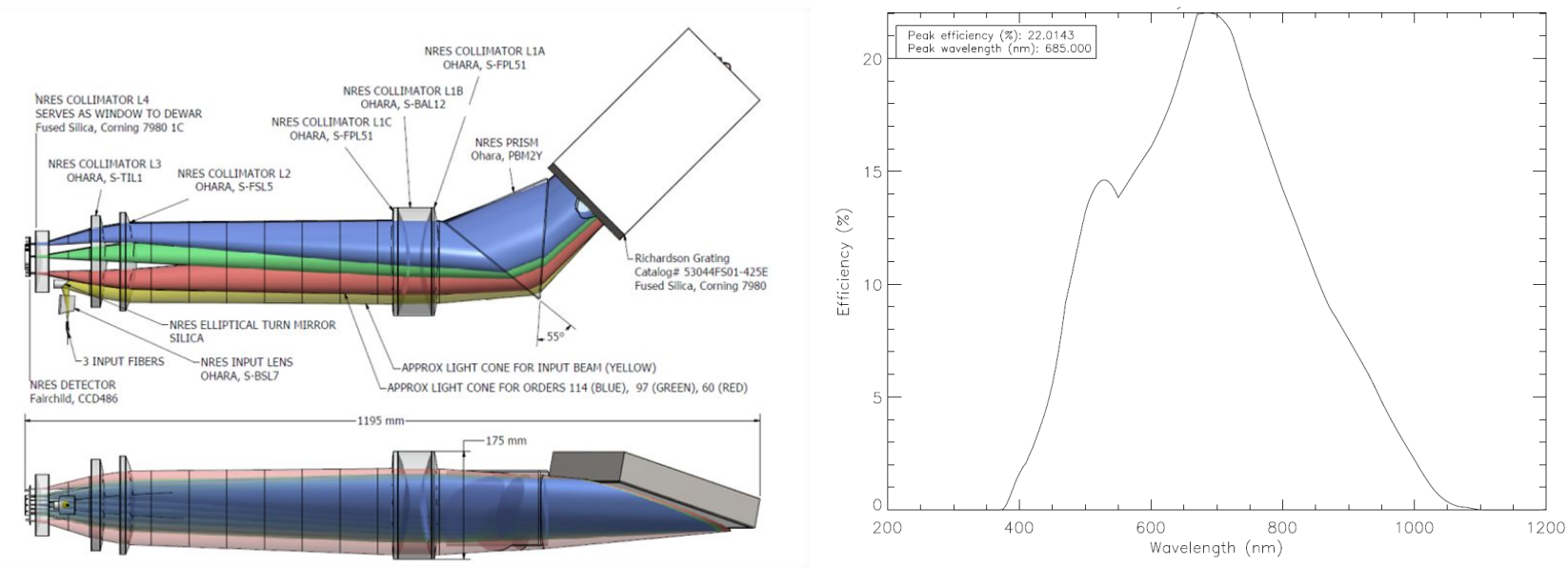

Figure 2. Left: NRES double-pass optical design schematic as seen from above (top) and side (bottom). After entering the instrument by fiber, light is directed by mirror into the collimator. The collimated light encounters the prism, grating, and prism again (cross-dispersed) before passing through the collimator a second time to focus light onto the detector via field flattener (far left). The relatively small number of surfaces helps maximize throughput. Right: expected total NRES system efficiency, including both spectrograph and telescope optics. System efficiency will be measured in the field once deployed to a telescope.
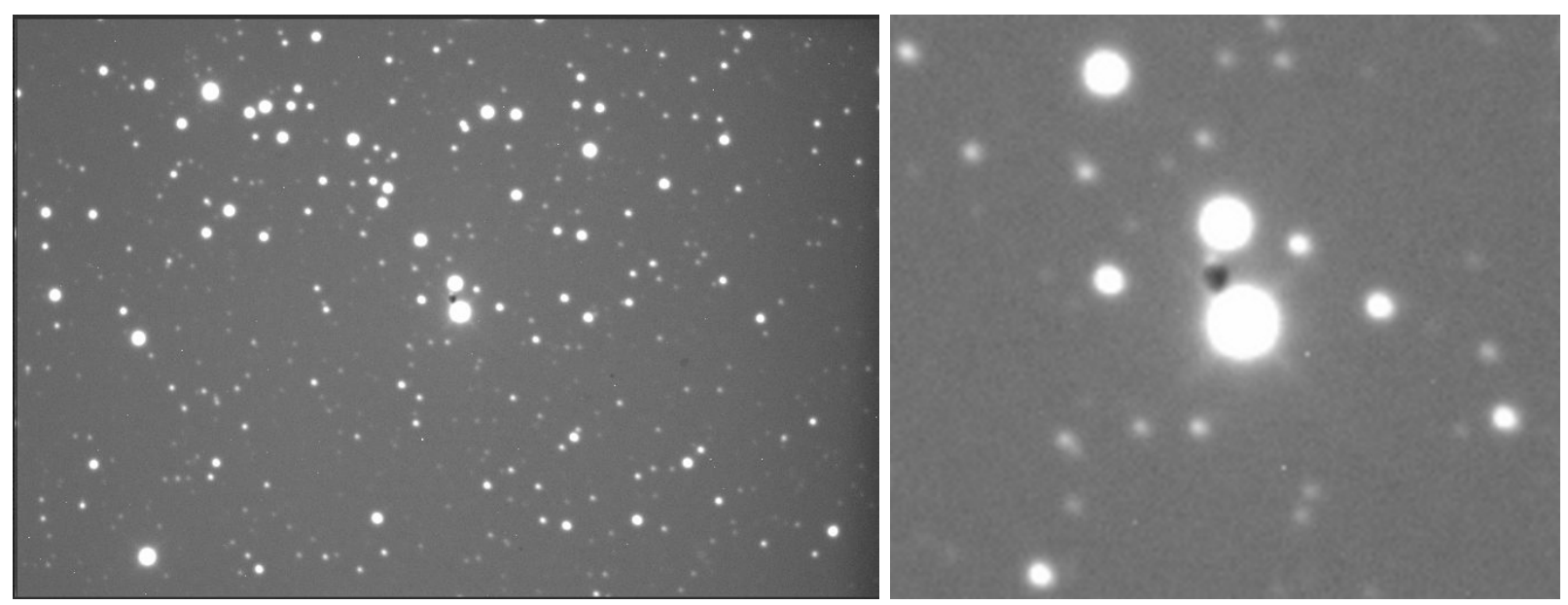

Figure 3. Left: example Acquisition and Guiding Unit (AGU) image from the NRES prototype spectrograph. The prototype field-of-view is roughly 14' x 9'. Right: enlarged cut-out of the image center that illustrates the pinhole (the black dot near image center) behind which the science fiber attaches. Light passing through that hole is re-imaged onto the fiber and sent to the spectrograph. Successful robotic acquisition and guiding require reliable, on-the-fly image analysis and coordinate solving. The prototype NRES spectrograph has been an extremely useful resource in the development of control software and new guide/acquisition algorithms.

required significant new software development and fine-tuning in order to work acceptably. The prototype will likely remain an important part of our development process until the entire NRES system is deployed.

\section{IGLOO AND ENVIRONMENTAL CONTROL}

To achieve the desired $3 \mathrm{~m} / \mathrm{s}$ precision level, an NRES spectrograph must maintain its internal temperature and pressure to $0.01 \mathrm{C}$ (RMS) and $0.02 \mathrm{mb}$ (RMS), respectively. We achieve this using a multi-stage system. Each NRES spectrograph is housed in its own enclosure called an "Igloo." The Igloo is a modified refrigerated reefer 


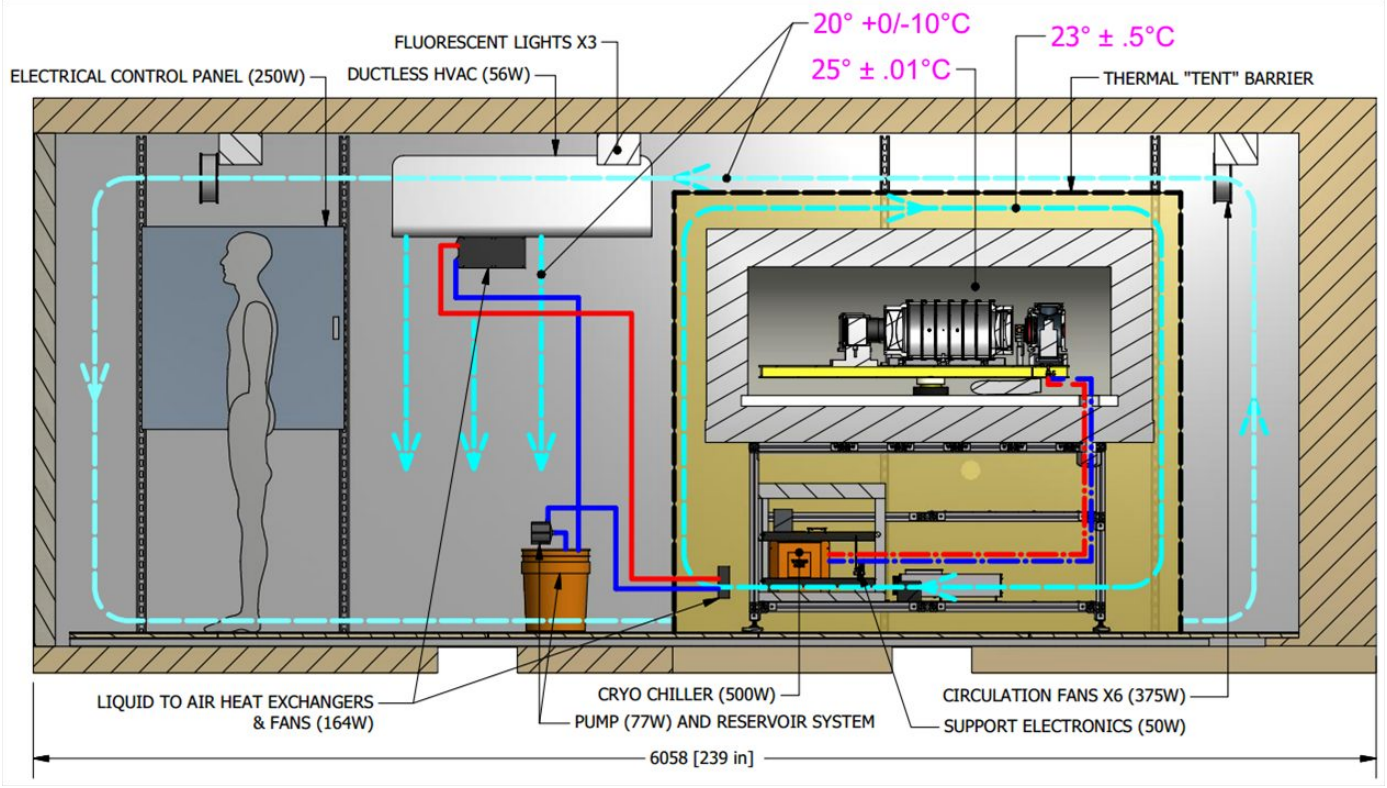

Figure 4. Schematic of the NRES "Igloo" enclosure that provides the needed environmental stability. Each NRES Igloo consists of a refrigerated reefer shipping container with a number of key modifications. The original heavy-duty HVAC is replaced with a ductless Mitsubishi model offering remote control plus heating in addition to cooling. High-speed fans at key locations are used to greatly increase the air circulation rate. Most importantly, we use a plastic thermal tent structure with servo-controlled intake and outflow fans to create an isolated room-within-a-room that reduces air temperature fluctuations surrounding the spectrograph by a factor of 5-10 more than what could be done with the outer A/C alone.

shipping container (inexpensive, well-insulated, and standardized) that provides the ambient stability necessary to reach our performance goal.

The reefer container is modified in a number of ways to improve its performance. In addition to extra insulation, we replace the oversized HVAC system with a smaller, ductless Mitsubishi (the same model already employed in our telescope domes) that can provide heating as well as cooling. High-speed fans placed in key positions are used to speed air circulation and further dampen temperature fluctuations. With fans assisting circulation, the Mitsubishi unit is able to maintain temperature to $\sim 1$ degree C RMS in the outer portion of the Igloo.

To further stabilize conditions, we erect an insulating plastic tent of inside the Igloo and around the spectrograph. The tent is equipped with intake and outflow fans which are actively controlled by a servo mechanism. This combination of fans and thermal barrier has outperformed its expectations, reducing temperature variation by a factor of 5 or 10 relative to the main compartment. This provides a thermally stable environment $(\sim 0.2$ degrees C RMS) in which we can install the instrument.

The spectrograph optics reside inside a double-walled, pressure-sealed, insulated chamber with a long thermal time constant. Further, the chamber wall layers contain sensors and heaters that actively minimize temperature gradients along the chamber. The optics chamber is fed a steady stream of dry air (inexpensive and readily available) at a slight positive pressure. This both stabilizes the environment and minimizes the possibility of particulate contamination. Combined with the already steady conditions provided by our thermal tent, we anticipate no difficulty reaching (or perhaps even exceeding) our target of $0.01 \mathrm{C}$.

\section{LAB TESTING}

For over a year, we have been operating the first NRES unit in a clean room environment using lamps and light from the daytime sky. Our sky fiber is a carefully-constructed, roof-mounted collecting system that mimics the 

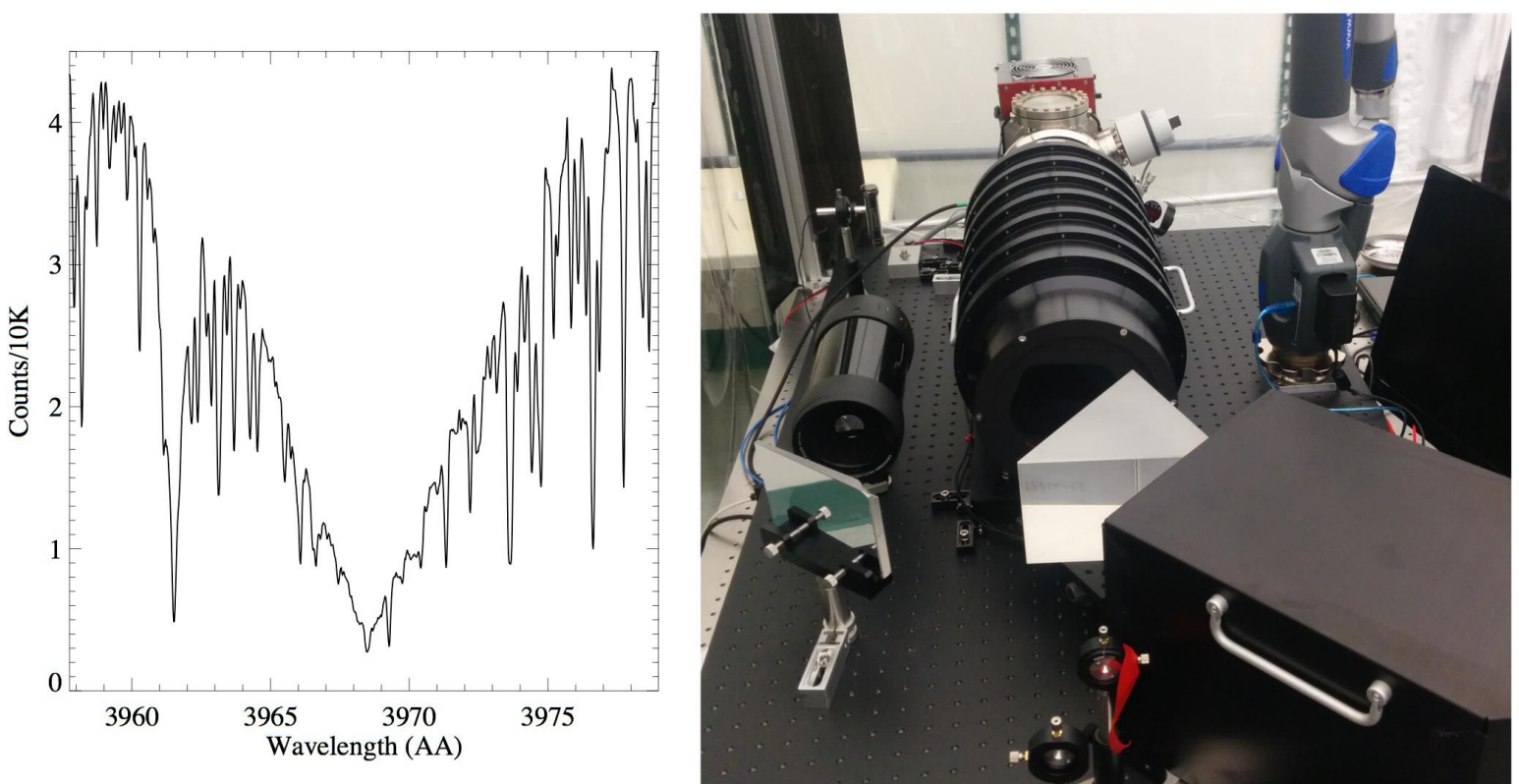

Figure 5. Left: extracted spectrum of Calcium II from the daytime sky acquired using the first production spectrograph during lab testing. Spectral resolution and optical throughput both agree well with the design. Right: photo of the lab setup used to obtain the measurement. The large black tube near image center is the collimator. The smaller black tube to the left is the spotting scope that collects light for the exposure meter.

light of a 4th magnitude star with the same focal ratio expected from the telescope + AGU system.

A photograph of the clean room instrument setup and a sample of extracted sky spectrum are shown in Figure 5. Testing indicates that the instrument performs as expected in resolution and throughput.

The daytime sky spectra have also provided valuable data for radial velocity pipeline development. As the Earth rotates, the apparent velocity of the observed patch of sky continuously changes at roughly $1.1 \mathrm{~m} / \mathrm{s}$ per minute. Our current pipline successfully recovers this signal with $\sim 25 \mathrm{~m} / \mathrm{s}$ RMS scatter, which is consistent with the amplitude of temperature swings experienced by the spectrograph. We expect most of this scatter to disappear in a more stable environment and expect to prove this in the coming months.

\section{ACKNOWLEDGMENTS}

Funding for the NRES spectrographs was provided through MRI Grant AST-1229720 and ATI grant AST1508464 from the National Science Foundation (NSF). We are grateful for their ongoing support.

\section{REFERENCES}

[1] Brown, T. M., Baliber, N., Bianco, F. B., Bowman, M., Burleson, B., Conway, P., Crellin, M., Depagne, É., De Vera, J., Dilday, B., Dragomir, D., Dubberley, M., Eastman, J. D., Elphick, M., Falarski, M., Foale, S., Ford, M., Fulton, B. J., Garza, J., Gomez, E. L., Graham, M., Greene, R., Haldeman, B., Hawkins, E., Haworth, B., Haynes, R., Hidas, M., Hjelstrom, A. E., Howell, D. A., Hygelund, J., Lister, T. A., Lobdill, R., Martinez, J., Mullins, D. S., Norbury, M., Parrent, J., Paulson, R., Petry, D. L., Pickles, A., Posner, V., Rosing, W. E., Ross, R., Sand, D. J., Saunders, E. S., Shobbrook, J., Shporer, A., Street, R. A., Thomas, D., Tsapras, Y., Tufts, J. R., Valenti, S., Vander Horst, K., Walker, Z., White, G., and Willis, M., "Las Cumbres Observatory Global Telescope Network," PASP 125, 1031-1055 (Sept. 2013).

[2] Volgenau, N. a., "Two years of LCOGT network operations: the challenges of a global observatory," in [Observatory Operations: Strategies, Processes, and Systems VI], Proc. SPIE 9910, 991050 (July 2016). 
[3] Akeson, R. L., Chen, X., Ciardi, D., Crane, M., Good, J., Harbut, M., Jackson, E., Kane, S. R., Laity, A. C., Leifer, S., Lynn, M., McElroy, D. L., Papin, M., Plavchan, P., Ramírez, S. V., Rey, R., von Braun, K., Wittman, M., Abajian, M., Ali, B., Beichman, C., Beekley, A., Berriman, G. B., Berukoff, S., Bryden, G., Chan, B., Groom, S., Lau, C., Payne, A. N., Regelson, M., Saucedo, M., Schmitz, M., Stauffer, J., Wyatt, P., and Zhang, A., "The NASA Exoplanet Archive: Data and Tools for Exoplanet Research," PASP 125, 989-999 (Aug. 2013).

[4] Collier Cameron, A., Bouchy, F., Hébrard, G., Maxted, P., Pollacco, D., Pont, F., Skillen, I., Smalley, B., Street, R. A., West, R. G., Wilson, D. M., Aigrain, S., Christian, D. J., Clarkson, W. I., Enoch, B., Evans, A., Fitzsimmons, A., Fleenor, M., Gillon, M., Haswell, C. A., Hebb, L., Hellier, C., Hodgkin, S. T., Horne, K., Irwin, J., Kane, S. R., Keenan, F. P., Loeillet, B., Lister, T. A., Mayor, M., Moutou, C., Norton, A. J., Osborne, J., Parley, N., Queloz, D., Ryans, R., Triaud, A. H. M. J., Udry, S., and Wheatley, P. J., "WASP1b and WASP-2b: two new transiting exoplanets detected with SuperWASP and SOPHIE," MNRAS 375, 951-957 (Mar. 2007).

[5] Bakos, G., Noyes, R. W., Kovács, G., Stanek, K. Z., Sasselov, D. D., and Domsa, I., "Wide-Field Millimagnitude Photometry with the HAT: A Tool for Extrasolar Planet Detection," PASP 116, 266-277 (Mar. 2004).

[6] Pepper, J., Pogge, R. W., DePoy, D. L., Marshall, J. L., Stanek, K. Z., Stutz, A. M., Poindexter, S., Siverd, R., O'Brien, T. P., Trueblood, M., and Trueblood, P., "The Kilodegree Extremely Little Telescope (KELT): A Small Robotic Telescope for Large-Area Synoptic Surveys," PASP 119, 923-935 (Aug. 2007).

[7] Ricker, G. R., Latham, D. W., Vanderspek, R. K., Ennico, K. A., Bakos, G., Brown, T. M., Burgasser, A. J., Charbonneau, D., Clampin, M., Deming, L. D., Doty, J. P., Dunham, E. W., Elliot, J. L., Holman, M. J., Ida, S., Jenkins, J. M., Jernigan, J. G., Kawai, N., Laughlin, G. P., Lissauer, J. J., Martel, F., Sasselov, D. D., Schingler, R. H., Seager, S., Torres, G., Udry, S., Villasenor, J. N., Winn, J. N., and Worden, S. P., "Transiting Exoplanet Survey Satellite (TESS)," in [American Astronomical Society Meeting Abstracts \#215], Bulletin of the American Astronomical Society 42, 459 (Jan. 2010).

[8] Eastman, J. D., Brown, T. M., Hygelund, J., van Eyken, J., Tufts, J. R., and Barnes, S., "NRES: the network of robotic Echelle spectrographs," in [Ground-based and Airborne Instrumentation for Astronomy V], Proc. SPIE 9147, 914716 (July 2014). 